\title{
A non-standard matter distribution in the RS1 model and the coupling constant of the radion
}

\author{
Mikhail N. Smolyakov, Igor P. Volobuev \\ Skobeltsyn Institute of Nuclear Physics, Moscow State University, \\ Vorob'evy Gory, 119992 Moscow, Russia
}

\begin{abstract}
In the zero mode approximation we solve exactly the equations of motion for linearized gravity in the Randall-Sundrum model with a non-standard distribution of matter in the neighbourhood of the negative tension brane. It is shown that the form of this distribution can strongly affect the coupling of the radion to matter. We believe that such a situation can arise in models with a realistic mechanisms of matter localization.
\end{abstract}

\section{Introduction}

The Kaluza-Klein hypothesis has been discussed in theoretical physics for three quarters of a century. In accordance with this hypothesis, space-time may have extra dimensions, which are unobservable for certain reasons. The explanation of this unobservability, which was put forward in the original papers by Kaluza and Klein, implies that the extra dimensions are compactified and have a very small size of the order of the Planck's length $l_{P l}=1 / M_{P l}$.

In 1983 Rubakov and Shaposhnikov put forward a new scenario for Kaluza-Klein theories, which was based on the idea of localization of fields on a domain wall [1]. They have also proposed an ansatz for multidimensional metric, which is compatible with this hypothesis [2].

In the last years there appeared indications that scenarios of this type can arise in the theory of strings [3, 4, 5, 6] (see Ref. [7, for a review and references). In this approach our three spatial dimensions are supposed to be realized as a three-dimensional hypersurface embedded into a mutidimensional space-time. Such hypersurfaces are called 3-branes, or just branes. The main goal of such scenarios was to find a solution to the hierarchy problem. It was solved either due to the sufficiently large characteristic size of extra dimensions [8], or due to exponential warp factor appearing in the metric [9]. In both approaches gravity in multidimensional space-time becomes "strong" not at the energies of the order of $10^{19} \mathrm{GeV}$, but at much lower energies, maybe of the order of $1 \div 10 \mathrm{TeV}$. An attractive feature of these models is that they predict new effects which can be observed at the coming collider experiments.

In paper [9] an exact solution for a system of two branes interacting with gravity in a five-dimensional space-time $E$ was found. This model is called the Randall-Sundrum model (usually abbreviated as RS1 model), and it is widely discussed in the literature (see Refs. [10. 11] for reviews and references). Let us denote the coordinates by $\left\{x^{M}\right\} \equiv\left\{x^{\mu}, y\right\}$, $M=0,1,2,3,4, \mu=0,1,2,3$, the coordinate $x^{4} \equiv y$ parameterizing the fifth dimension. It forms the orbifold $S^{1} / Z_{2}$, which is realised as the circle of the circumference $2 R$ with points $y$ and $-y$ identified. Correspondingly, we have the usual periodicity condition in space-time 
$E$, which identifies points $(x, y)$ and $(x, y+2 n R)$, and the metric $g_{M N}$ satisfies the orbifold symmetry conditions

$$
\begin{array}{r}
g_{\mu \nu}(x,-y)=g_{\mu \nu}(x, y), \\
g_{\mu 4}(x,-y)=-g_{\mu 4}(x, y), \\
g_{44}(x,-y)=g_{44}(x, y) .
\end{array}
$$

The branes are located at the fixed points of the orbifold, $y=0$ and $y=R$.

The action of the model is

$$
S=S_{g}+S_{1}+S_{2}
$$

where $S_{g}, S_{1}$ and $S_{2}$ are given by

$$
\begin{aligned}
S_{g} & =\frac{1}{16 \pi \hat{G}} \int_{E}(R-\Lambda) \sqrt{-g} d^{4} x d y, \\
S_{1} & =V_{1} \int_{E} \sqrt{-\tilde{g}} \delta(y) d^{4} x d y, \\
S_{2} & =V_{2} \int_{E} \sqrt{-\tilde{g}} \delta(y-R) d^{4} x d y .
\end{aligned}
$$

Here $\tilde{g}_{\mu \nu}$ is the induced metric on the branes and the subscripts 1 and 2 label the branes. We also note that the signature of the metric $g_{M N}$ is chosen to be $(-,+,+,+,+)$.

The Randall-Sundrum solution for the metric is given by

$$
d s^{2}=g_{M N} d x^{M} d x^{N}=\gamma_{\mu \nu} d x^{\mu} d x^{\nu}+d y^{2},
$$

where $\gamma_{\mu \nu}=e^{2 \sigma(y)} \eta_{\mu \nu}, \eta_{\mu \nu}$ is the Minkowski metric and the function $\sigma(y)=-k|y|$ in the interval $-R \leq y \leq R$. The parameter $k$ is positive and has the dimension of mass, the parameters $\Lambda$ and $V_{1,2}$ are related to it as follows:

$$
\Lambda=-12 k^{2}, \quad V_{1}=-V_{2}=-\frac{3 k}{4 \pi \hat{G}} .
$$

We see that brane 1 has a positive energy density, whereas brane 2 has a negative one. The function $\sigma$ has the properties

$$
\partial_{4} \sigma=-k \operatorname{sign}(y), \quad \frac{\partial^{2} \sigma}{\partial y^{2}}=-2 k(\delta(y)-\delta(y-R)) \equiv-2 k \tilde{\delta} .
$$

Here and in the sequel $\partial_{4} \equiv \frac{\partial}{\partial y}$.

We denote $\hat{\kappa}=\sqrt{16 \pi \hat{G}}$, where $\hat{G}$ is the five-dimensional gravitational constant, and parameterize the metric $g_{M N}$ as

$$
g_{M N}=\gamma_{M N}+\hat{\kappa} h_{M N}
$$

$h_{M N}$ being the metric fluctuations. Substituting this parameterization into (2) and retaining the terms of the zero order in $\hat{\kappa}$, we get the second variation action of this model [12. It is invariant under the gauge transformations

$$
h_{M N}^{\prime}(x, y)=h_{M N}(x, y)-\left(\nabla_{M} \xi_{N}(x, y)+\nabla_{N} \xi_{M}(x, y)\right)
$$


where $\nabla_{M}$ is the covariant derivative with respect to the background metric $\gamma_{M N}$, and the functions $\xi_{N}(x, y)$ satisfy the orbifold symmetry conditions

$$
\begin{aligned}
& \xi^{\mu}(x,-y)=\xi^{\mu}(x, y), \\
& \xi^{4}(x,-y)=-\xi^{4}(x, y) .
\end{aligned}
$$

With the help of these gauge transformations we can impose the gauge

$$
h_{\mu 4}=0, h_{44}=h_{44}(x) \equiv \phi(x),
$$

which will be called the unitary gauge (see [12]). We would like to emphasize once again that the branes remain straight in this gauge, i.e. we do not use the bent-brane formulation, which allegedly destroys the structure of the model, i.e. the orbifold symmetry under the reflection $-y \leftrightarrow y$ (this problem was discussed in [13]).

In the linear approximation interaction with matter looks like

$$
\frac{\hat{\kappa}}{2} \int h^{M N}(x, y) T_{M N} \sqrt{-\gamma} d^{4} x d y
$$

where $T_{M N}$ is the energy-momentum tensor of the matter:

$$
T_{M N}=2 \frac{\delta L}{\delta \gamma^{M N}}-\gamma_{M N} L
$$

Thus, we are considering perturbations about the background, for which $T_{M N}=0$.

We will examine the $h_{\mu \nu}$-components of the metric fluctuations $h_{M N}$, since all fourdimensional physical effects can be described in terms of this field. Obviously, the unitary gauge conditions (9) do not fix the gauge of this field. In fact, after imposing these gauge conditions there remain gauge transformations of the form

$$
\xi_{\mu}=e^{2 \sigma} \epsilon_{\mu}(x)
$$

which change the longitudinal components of the field $h_{\mu \nu}$. Nevertheless, it turns out that it is convenient to solve the equations of motion for linearized gravity in the unitary gauge and then to choose an appropriate gauge in our four-dimensional world on the brane. We will use the de Donder gauge for the field $h_{\mu \nu}$ on the brane, which corresponds to the choice of harmonic coordinates.

\section{Equations of motion}

The equations of motion for different components of the metric fluctuations in the unitary gauge take the form (see [14, 15]):

1) $\mu \nu$-component

$$
\begin{aligned}
& \frac{1}{2}\left(\partial_{\rho} \partial^{\rho} h_{\mu \nu}-\partial_{\mu} \partial^{\rho} h_{\rho \nu}-\partial_{\nu} \partial^{\rho} h_{\rho \mu}+\frac{\partial^{2} h_{\mu \nu}}{\partial x^{4^{2}}}\right)-2 k^{2} h_{\mu \nu}+\frac{1}{2} \partial_{\mu} \partial_{\nu} \tilde{h}+ \\
+ & \frac{1}{2} \partial_{\mu} \partial_{\nu} \phi+\frac{1}{2} \gamma_{\mu \nu}\left(\partial^{\rho} \partial^{\sigma} h_{\rho \sigma}-\partial_{\rho} \partial^{\rho} \tilde{h}-\frac{\partial^{2} \tilde{h}}{\partial x^{4^{2}}}-4 \partial_{4} \sigma \partial_{4} \tilde{h}-\partial_{\rho} \partial^{\rho} \phi+12 k^{2} \phi\right)+ \\
+ & {\left[2 k h_{\mu \nu}-3 k \gamma_{\mu \nu} \phi\right] \tilde{\delta}=-\frac{\hat{\kappa}}{2} T_{\mu \nu} }
\end{aligned}
$$


2) $\mu 4$-component,

$$
\partial_{4}\left(\partial_{\mu} \tilde{h}-\partial^{\nu} h_{\mu \nu}\right)-3 \partial_{4} \sigma \partial_{\mu} \phi=-\hat{\kappa} T_{\mu 4},
$$

which plays the role of a constraint,

3) 44-component

$$
\frac{1}{2}\left(\partial^{\mu} \partial^{\nu} h_{\mu \nu}-\partial_{\mu} \partial^{\mu} \tilde{h}\right)-\frac{3}{2} \partial_{4} \sigma \partial_{4} \tilde{h}+6 k^{2} \phi=-\frac{\hat{\kappa}}{2} T_{44}
$$

with $T_{M N}$ being the energy-momentum tensor of the matter and $\tilde{h}=\gamma^{\mu \nu} h_{\mu \nu}$. In what follows, we will also use an auxiliary equation, which is obtained by multiplying the equation for 44component by 2 and subtracting it from the contracted equation for $\mu \nu$-component. This equation contains $\tilde{h}$ and $\phi$ only and has the form:

$$
\frac{\partial^{2} \tilde{h}}{\partial x^{4^{2}}}+2 \partial_{4} \sigma \partial_{4} \tilde{h}-8 k^{2} \phi+8 k \phi \tilde{\delta}+\partial_{\mu} \partial^{\mu} \phi=\frac{\hat{\kappa}}{3}\left(T_{\mu}^{\mu}-2 T_{44}\right) .
$$

For example, if $T_{M N}=0$, the physical degrees of freedom of the model can be extracted by the substitution 12

$$
h_{\mu \nu}=b_{\mu \nu}+\gamma_{\mu \nu}(\sigma-c) \phi+\frac{1}{2 k^{2}}\left(\sigma-c+\frac{1}{2}+\frac{c}{2} e^{-2 \sigma}\right) \partial_{\mu} \partial_{\nu} \phi .
$$

with $c=\frac{k R}{e^{2 k R}-1}$. It turns out that the field $b_{\mu \nu}\left(x^{\mu}, y\right)$ describes the massless graviton and massive Kaluza-Klein spin-2 fields, whereas $\phi(x)$ describes a scalar field called the radion.

However, the situation is rather different, when there is matter on the branes. The cases, in which matter is located on the branes, i.e. the energy-momentum tensor is of the form $T_{\mu \nu}=t_{\mu \nu}(x) \delta(y)$ or $T_{\mu \nu}=t_{\mu \nu}(x) \delta(y-R)$ (and $T_{\mu 4}=0, T_{44}=0$ ) were discussed in detail in [14, 15. It is well known that if we live on the negative tension brane (at $y=R$ ), the contribution of the radion is $e^{2 k R}$ times stronger than the contribution of the massless graviton [14, 15]. It means that in the case of the massless radion scalar gravity is realized on brane 2 , and it is necessary to have a mechanism for generating the radion mass, for example, the Goldberger-Wise mechanism [16] to make gravity in the zero mode approximation tensor, i.e. to suppress the contribution of the scalar radion component. But we will show below, that this is not the only solution for this problem.

\section{A simple example}

In the original formulation of the Randall-Sundrum model the mechanism of localization of fields is not taken into account. The branes are treated as infinitely thin objects, and it is assumed that energy-momentum tensor has $\delta(y)$-like profile in the extra coordinate (matter is located on the brane only). Nevertheless in some papers (see, for example, 17, 18, 19, 20, 21]) considering models with extra dimensions, energy-momentum tensors with dependence on the extra coordinates were used for constructing non-trivial background metric. One can also recall models with universal extra dimensions, in which physical fields can propagate in the extra dimension [22, and fat brane scenarios [23, 24]. Thus, the existence of energymomentum tensors of this type seems to be quite reasonable. Here we consider some simple 
examples, which are sufficient to show attractive features of the non-delta-like localization of matter.

Let us choose as an example the following form of the energy-momentum tensor

$$
\begin{aligned}
T_{\mu \nu} & =t_{\mu \nu}(x) \frac{e^{-2 \sigma} c}{R}, \\
T_{\mu 4} & \equiv 0, \\
T_{44} & =t_{\mu \nu}(x) \gamma^{\mu \nu} e^{-2 \sigma} \frac{c}{R}\left(\sigma+e^{2 k R} c-\epsilon c\right),
\end{aligned}
$$

where $\epsilon$ is some arbitrary constant, which will be defined later. Here $t_{\mu \nu}(x)$ is the energymomentum tensor of matter on the brane, it depends on the four-dimensional coordinates $x$ only. One can see that the matter is localized near the negative tension brane at $y=R$. We would like to note that the energy-momentum tensor of the form (17), (18) and (19) satisfies the covariant energy conservation law, which has the form

$$
\nabla^{N} T_{M N}=0
$$

and the function of localization in (17) is normalized to unity. Constructions similar to that used in this paper, but in the case of six dimensions, were utilized in [25, 26]. The explanation of such constructions depends on the method of localization, and this issue will not be discussed in this paper.

The substitution, which allows one to decouple the equations (12), (13), (14), (15) with $T_{M N}$ of the form (17), (18), (19) looks like

$$
b_{\mu \nu}=u_{\mu \nu}+\frac{1}{2 k^{2}}\left(\frac{c e^{2 k R}}{\epsilon}-e^{-2 \sigma}\left[\frac{c e^{2 k R}}{2 \epsilon}+\frac{1}{8 \epsilon}\right]-\sigma e^{-2 \sigma} \frac{1}{2 \epsilon}\right) \partial_{\mu} \partial_{\nu} \phi,
$$

where $b_{\mu \nu}$ was defined in (16) and $u_{\mu \nu}\left(x^{\mu}, y\right)$ describes the massless graviton and massive Kaluza-Klein spin-2 fields. Substituting (16) and (21) into equations (13), (14) and (15), we get:

$$
\begin{gathered}
\partial_{4}\left(e^{-2 \sigma}\left(\partial^{\nu} u_{\mu \nu}-\partial_{\mu} u\right)\right)=0, \\
e^{-4 \sigma}\left(\partial^{\mu} \partial^{\nu} u_{\mu \nu}-\square u\right)-3 \partial_{4} \sigma \partial_{4}\left(e^{-2 \sigma} u\right)+e^{-2 \sigma} \frac{3 c e^{2 k R}}{\epsilon} \square \phi- \\
-\frac{3}{\epsilon} e^{-4 \sigma}\left(\sigma+\tilde{c} e^{2 k R}\right) \square \phi=-\hat{\kappa} t \frac{c}{R} e^{-4 \sigma}\left(\sigma+\tilde{c} e^{2 k R}\right), \\
\partial_{4}\left(e^{2 \sigma} \partial_{4}\left(e^{-2 \sigma} u\right)\right)+\frac{1}{\epsilon} \square \phi e^{-2 \sigma}\left(1-2\left(\sigma+\tilde{c} e^{2 k R}\right)\right)= \\
=\frac{\hat{\kappa} c}{3 R} t e^{-2 \sigma}\left(1-2\left(\sigma+\tilde{c} e^{2 k R}\right)\right),
\end{gathered}
$$

where $u=\eta^{\mu \nu} u_{\mu \nu}, t=\eta^{\mu \nu} t_{\mu \nu}, \partial^{\mu}=\eta^{\mu \nu} \partial_{\nu}, \square=\eta^{\mu \nu} \partial_{\mu} \partial_{\nu}$ and

$$
\tilde{c}=c\left(1-\epsilon e^{-2 k R}\right)
$$


Let us consider the Fourier expansion of all terms of equation (24) with respect to coordinate $y$. Since the term with the derivative $\partial_{4}$ has no zero mode, this equation implies that

$$
\begin{gathered}
\square \phi=\frac{\hat{\kappa} \epsilon c}{3 R} t, \\
\partial_{4}\left(e^{-2 \sigma} u\right)=0 .
\end{gathered}
$$

Now let us consider $\mu \nu$-equation. It is well known that the field $u_{\mu \nu}$ in the presence of matter is a combination of zero and massive modes, whose eigenfunctions are orthogonal [12. In particular, the zero mode can be represented as $u_{\mu \nu}^{0}=e^{2 \sigma} \alpha_{\mu \nu}$, where $\alpha_{\mu \nu}$ depends on $x$ only. It also means that with the help of the residual gauge transformations (11) it is possible to impose the gauge condition

$$
\begin{aligned}
\partial^{\nu} u_{\mu \nu}^{m} & =0, \\
u_{\mu}^{m \mu} & =0,
\end{aligned}
$$

on the massive modes $u_{\mu \nu}^{m}$ and the de Donder gauge condition on the zero mode

$$
\partial^{\nu}\left(\alpha_{\mu \nu}-\frac{1}{2} \eta_{\mu \nu} \alpha\right)=0 .
$$

Having imposed this gauge, we are still left with residual gauge transformation

$$
\xi_{\mu}=e^{2 \sigma} \epsilon_{\mu}(x), \quad \square \epsilon_{\mu}=0 .
$$

The gauge transformations with $\xi_{\mu}$ satisfying these conditions are important for determining the number of degrees of freedom of the massless graviton. It follows from equation (23) that

$$
\square \alpha=2 \frac{\hat{\kappa} e^{2 k R} c^{2}}{R} t
$$

Substituting (16) and (21) into equation (12) with condition (25) and passing to gauge (27), (28) we get

$$
\begin{array}{r}
\frac{1}{2} \square\left(\alpha_{\mu \nu}-\frac{1}{2} \eta_{\mu \nu} \alpha\right)+\frac{1}{2} e^{-2 \sigma} \square u_{\mu \nu}^{m}+\frac{1}{2} \partial_{4} \partial_{4} u_{\mu \nu}^{m}-2 k^{2} u_{\mu \nu}^{m}-\partial_{4} \partial_{4} \sigma u_{\mu \nu}^{m}= \\
=-\frac{\hat{\kappa} c}{2 R} t_{\mu \nu} e^{-2 \sigma}+\frac{\hat{\kappa} c}{6 R}\left(2 c e^{2 k R}-e^{-2 \sigma}\right)\left(\frac{\partial_{\mu} \partial_{\nu}}{\square}-\eta_{\mu \nu}\right) t
\end{array}
$$

We are going to calculate the equations of motion in the zero mode approximation. Thus, we have to find an equation for the field $\alpha_{\mu \nu}$. If we multiply equation (31) by $e^{2 \sigma}$, integrate it over $y$ and take into account the orthonormality condition for the wave functions of the modes [12, we get

$$
\square\left(\alpha_{\mu \nu}-\frac{1}{2} \eta_{\mu \nu} \alpha\right)=-2 \frac{\hat{\kappa} c^{2} e^{2 k R}}{R} t_{\mu \nu} .
$$

The equation for massive modes takes the form

$$
\begin{array}{r}
\frac{1}{2} e^{-2 \sigma} \square u_{\mu \nu}^{m}+\frac{1}{2} \partial_{4} \partial_{4} u_{\mu \nu}^{m}-2 k^{2} u_{\mu \nu}^{m}-\partial_{4} \partial_{4} \sigma u_{\mu \nu}^{m}= \\
=-\frac{\hat{\kappa} c}{2 R} t_{\mu \nu}\left(e^{-2 \sigma}-2 c e^{2 k R}\right)+\frac{\hat{\kappa} c}{6 R}\left(2 c e^{2 k R}-e^{-2 \sigma}\right)\left(\frac{\partial_{\mu} \partial_{\nu}}{\square}-\eta_{\mu \nu}\right) t
\end{array}
$$


Now we are ready to find equations of motion for the zero mode part of $h_{\mu \nu}$. Since the matter is localized near the brane, we will calculate the equation for $\left.h_{\mu \nu}\right|_{y=R}$. It is easy to see that $\left.h_{\mu \nu}\right|_{y=R}$ (16), (21) does not satisfy the de Donder gauge condition. The residual gauge transformations (29) are not sufficient to pass to this gauge. But since we consider only the effective theory on brane 2 (at $y=R$ ), we can drop the condition $\square \epsilon_{\mu}=0$, which fixes the gauge for the field $h_{\mu \nu}$. Then we can pass to the de Donder gauge condition for the field $h_{\mu \nu}$ on brane 2 with the help of these gauge functions. Making these transformations (analogously to what was made in [14, 15]), we get in the zero mode approximation

$$
\begin{array}{r}
\left.\left(h_{\mu \nu}-\frac{1}{2} \eta_{\mu \nu} h\right)\right|_{y=R}= \\
=e^{-2 k R}\left(\alpha_{\mu \nu}-\frac{1}{2} \eta_{\mu \nu} \alpha\right)-2 e^{-2 k R}(-k R-c)\left(\eta_{\mu \nu}-\frac{\partial_{\mu} \partial_{\nu}}{\square}\right) \phi .
\end{array}
$$

An important point is that these equations are written in the coordinates $\left\{x^{\mu}\right\}$, which are Galilean on brane 1 (not on brane 2) and are inappropriate for studying physical effects on brane 2 (we recall that coordinates are called Galilean, if $g_{\mu \nu}=\operatorname{diag}(-1,1,1,1)[27$ ). It is necessary to pass to Galilean coordinates on brane 2 to get a correct result. This problem was discussed in detail in papers [12, 14, 15. In Galilean coordinates on brane 2 equation (34) looks like

$$
\begin{array}{r}
\left.\left(h_{\mu \nu}-\frac{1}{2} \eta_{\mu \nu} h\right)\right|_{y=R}= \\
=e^{-2 k R}\left(\alpha_{\mu \nu}-\frac{1}{2} \eta_{\mu \nu} \alpha\right)-2(-k R-c)\left(\eta_{\mu \nu}-\frac{\partial_{\mu} \partial_{\nu}}{\square}\right) \phi,
\end{array}
$$

and equations (25), (32) take the form

$$
\begin{gathered}
\square \phi=\frac{\hat{\kappa} \epsilon c}{3 R} t, \\
\square\left(\alpha_{\mu \nu}-\frac{1}{2} \eta_{\mu \nu} \alpha\right)=-2 \frac{\hat{\kappa} c^{2} e^{4 k R}}{R} t_{\mu \nu} .
\end{gathered}
$$

Thus, we can get

$$
\left.\square\left(h_{\mu \nu}-\frac{1}{2} \eta_{\mu \nu} h\right)\right|_{y=R}=-\frac{2 \hat{\kappa} c^{2} e^{2 k R}}{R}\left(t_{\mu \nu}-\frac{\epsilon}{3}\left(\eta_{\mu \nu}-\frac{\partial_{\mu} \partial_{\nu}}{\square}\right) t\right),
$$

where $t_{\mu \nu}$ is the energy-momentum tensor of matter in the coordinates, which are Galilean at $y=R$. One can see, that this equation coincides with equation for the fluctuations of metric in the linearized Brans-Dicke theory, which looks like

$$
\square\left(\delta g_{\mu \nu}-\frac{1}{2} \eta_{\mu \nu} \delta g\right)=-16 \pi G\left(t_{\mu \nu}-\frac{1}{2 \omega+3}\left(\eta_{\mu \nu}-\frac{\partial_{\mu} \partial_{\nu}}{\square}\right) t\right),
$$

where $\omega$ is the BD-parameter and $G$ is the gravitational constant. Thus, comparing equations (38) and (39), we get

$$
G=\hat{G} \frac{2 c^{2} e^{2 k R}}{R}
$$




$$
\omega=\frac{3(1-\epsilon)}{2 \epsilon} .
$$

It is easy to see, that in the the Randall-Sundrum model with the $\delta$-function-like localization of matter on the brane there is a factor $e^{2 k R}$ instead of $\epsilon$ in (38) (compare with the analogous formula in [14, 15]).

From the recent experimental data (see, for example, [28] ) we know, that $\omega>3500$. It means, that

$$
\epsilon<4,3 \cdot 10^{-4}
$$

With this value of $\epsilon$ there are already no problems with the radion field.

\section{Localization of matter}

In (17) the factor $e^{-2 \sigma}$, describing distribution of matter in the extra dimension, was utilized. However one can say that matter is spread in the whole bulk rather than localized on the brane (in analogy to the massless graviton whose wave function is $\sim e^{2 \sigma}$ ), and it is not evident, why we make all calculations for $h_{\mu \nu}$ at $y=R$. It seems that it is more reasonable to consider matter, which is confined to brane 2 much stronger, since the description of gravity on brane 2 by $\left.h_{\mu \nu}\right|_{y=R}$ is more justified in this case. Let us consider a "toy model" with energy-momentum tensor of the form

$$
T_{\mu \nu}=t_{\mu \nu}(x) \frac{k(1+N)}{e^{2 k R+2 N k R}-1} e^{-2 \sigma(1+N)},
$$

where the function of localization is normalized to unity as well. Other components of the five-dimensional energy-momentum tensor are chosen to be

$$
\begin{gathered}
T_{\mu 4}=0 \\
T_{44}=t(x)(k+k N) e^{-4 \sigma}\left(\frac{c}{2 N k R}-\right. \\
\left.-\frac{c \epsilon e^{2 N k R}}{e^{2 k R+2 N k R}-1}-\frac{k}{2 N k\left(e^{2 k R+2 N k R}-1\right)} e^{-2 N \sigma}\right) .
\end{gathered}
$$

Such a complicated form of (45) is caused by the requirements to satisfy the energy conservation law (20) and to get a weak coupling constant for the radion.

The substitution, which allows one to decouple and solve equations (13), (14) and $\mu \nu$ equation with (43), (44) and (45), has the following form:

$$
\begin{array}{r}
b_{\mu \nu}=u_{\mu \nu}+\left(\frac{e^{2 k R}\left(1-e^{-2 N k R}\right)}{4 k^{2} N \epsilon\left(e^{2 k R}-1\right)}-\frac{\left(e^{2 k R}-e^{-2 N k R}\right)}{8 k^{2} N \epsilon\left(e^{2 k R}-1\right)} e^{-2 \sigma}+\right. \\
\left.+\frac{e^{-2 N k R}}{4 N k(N k+2 k) \epsilon} e^{-2 \sigma-2 N \sigma}\right) \partial_{\mu} \partial_{\nu} \phi
\end{array}
$$

Analogously to what was made in Section 3, we can get

$$
\square \phi=\frac{\hat{\kappa} \epsilon(k+k N) e^{2 N k R}}{3\left(e^{2 k R+2 N k R}-1\right)} t,
$$




$$
\square\left(\alpha_{\mu \nu}-\frac{1}{2} \eta_{\mu \nu} \alpha\right)=-\frac{\hat{\kappa} k(k+N k)\left(1-e^{-2 N k R}\right)}{N k\left(e^{2 k R}-1\right)\left(1-e^{-2 k R-2 N k R}\right)} t_{\mu \nu} .
$$

Using (46) and passing to Galilean coordinates at $y=R$, in the zero mode approximation we get

$$
\begin{array}{r}
\left.\square\left(h_{\mu \nu}-\frac{1}{2} \eta_{\mu \nu} h\right)\right|_{y=R}= \\
=-\frac{\hat{\kappa} c(1+N)\left(1-e^{-2 N k R}\right)}{N R\left(1-e^{-2 k R-2 N k R}\right)}\left(t_{\mu \nu}-\frac{2 N k R \epsilon}{3\left(1-e^{-2 N k R}\right)}\left(\eta_{\mu \nu}-\frac{\partial_{\mu} \partial_{\nu}}{\square}\right) t\right) .
\end{array}
$$

One can see that in the limit $N \rightarrow 0$ equation (49) passes into (38). The four-dimensional gravitational constant has the form

$$
G=\hat{G} \frac{c(1+N)\left(1-e^{-2 N k R}\right)}{N R\left(1-e^{-2 k R-2 N k R}\right)}
$$

(compare with (39)). For a large $N$ we get

$$
G \approx \hat{G} \frac{c}{R} .
$$

A choice of relatively small $\epsilon$ makes the contribution of the radion to be not in contradiction with the present-day experimental data.

\section{Conclusion and final remarks}

There may arise a question about generation of the new hierarchy instead of the one solved in the Randall-Sundrum model. Actually, the factor $\epsilon$ in (19) is much smaller than the factor $e^{2 k R}$ in (19). But for the energy-momentum tensor on brane $2($ at $y=R)$ we get

$$
\begin{array}{r}
\left.T_{\mu \nu}\right|_{y=R}=t_{\mu \nu} \frac{e^{2 k R} c}{R} \approx k t_{\mu \nu}, \\
\left.T_{44}\right|_{y=R}=t \frac{e^{4 k R} c^{2}}{R}\left(1-4.3 \cdot 10^{-4}\right) \approx t k^{2} R\left(1-4.3 \cdot 10^{-4}\right),
\end{array}
$$

and the correction $\sim \epsilon$ is not so small in comparison with the main value $\sim 1$ (analogous calculations can be made in the case (43) and (45) too). It seems to be quite reasonable. One should take into account that the energy-momentum tensors of the form (17), (18), (19) and (43), (44), (45) do not correspond to any real field action, and must be interpreted as "toy models". There also arises the question about the origin of such correction $\sim \epsilon$. Explicitly the term $\sim e^{-4 \sigma}$ in (19) and (45) corresponds to the homogeneous solution of equation (20) (i.e. to the case of $T_{\mu \nu}=0$ ). Nevertheless such term can be taken into consideration, since its existence does not evidently contradict our assumptions. Surely it would be better to derive similar form of the energy-momentum tensor in a more natural way. For example, simple examples with scalar and gauge fields in the bulk in the RandallSundrum model were discussed in papers [29, 30]. Wave functions of these fields have their 
maximum values on brane 2, and these solutions can be treated as a basis for our choice of the energy-momentum tensor. But even in the simplest case of the scalar field only, there arises a nonzero $T_{\mu 4}$-component of the energy-momentum tensor, which does not satisfy the condition (18). Moreover, the wave functions are different not only for different fields, but even for different modes. Thus, formulas (17), (18), (19) and (43), (44), (45) must be used as "toy models" for classical objects, but not for the fields (for example, one can choose $t_{00} \sim m \delta(\vec{x}), t_{i 0}=0, t_{i j}=0$ for a static point-like source).

In this paper we have discussed only one aspect of the radion field problem. Indeed, though the coupling constant of the radion to matter can be small enough to fit the experimental data, the size of extra dimension in the RS1 model should be stabilized in any case. It is well known that in this model gravity alone cannot stabilize the size of extra dimension due to the Casimir effect 31. But if matter is not localized exactly on the brane, i.e. it has wave functions in the extra dimension, it is possible that the contribution of matter fields to the Casimir force between two branes could stabilize the size of the extra dimension. Moreover, even in the case of classical stabilization with the help of scalar field (such as in 32 ) the coupling constant of the massive radion to matter can vary essentially depending on the profile of matter in the extra dimension.

There is another question, which may arise in connection with our choice for matter distribution: why do we choose non-delta-like profile for matter, whereas the branes have delta-like profile? Of course, such profile of the branes is an idealization. But one can recall kink-like solution found in [33], which describes a delta-like brane in the thin brane limit. Thus in the case under consideration the RS1 setup can be regarded as a phenomenological model for describing the branes in the case when the thickness of branes is much smaller than the effective width of the wave functions of matter. Another advantage of the RS1 background is its relative simplicity, which allowed us to decouple and solve exactly equations of motion for the radion field and tensor modes at least in the zero mode approximation and to show possible effects which can be produced by a non-standard matter distribution. In a more realistic model the non-zero width of the branes should be definitely taken into account, but it is evident that effect analogous to that described above should exist in this case too.

It should be also noted that the longitudinal parts of substitutions (16), (21) and (46) contain some terms, which can lead (but it is not necessarily so) to the strong coupling effect, analogously to what happens in the DGP model [34, 35]. These terms are pure gauge from the four-dimensional point of view from the brane and are not dangerous in the linear order. Nevertheless it is necessary to consider the non-linear corrections to the equations of motion to discover the contribution of these longitudinal terms. But this problem deserves an additional detailed investigation.

\section{Acknowledgments}

The authors are grateful to Yu.A. Kubyshin for valuable discussions. The work was supported by the RFBR grants 04-02-16476 and 04-02-17448, by the grant UR.02.02.503 of the scientific program "Universities of Russia", and by the grant NS.1685.2003.2 of the Russian Federal Agency for Science. 


\section{References}

[1] V.A. Rubakov, M.E. Shaposhnikov, Phys.Lett. B125 (1983) 136

[2] V.A. Rubakov, M.E. Shaposhnikov, Phys.Lett. B125 (1983) 139

[3] J. Polchinski, arXiv:hep-th/9611050

[4] E. Witten, Nucl.Phys. B471 (1996) 135

[5] J. Lykken, Phys.Rev. D54 (1996) 3693

[6] C.P. Bachas, arXiv: hep-th/9806199

[7] I. Antoniadis, arXiv: hep-th/0102202

[8] N. Arkani-Hamed, S. Dimopoulos, G. Dvali, Phys.Lett. B429 (1998) 263

[9] L. Randall, R. Sundrum, Phys.Rev.Lett. 83 (1999) 3370

[10] V.A. Rubakov, Phys.Usp. 44 (2001) 871

[11] Y.A. Kubyshin, arXiv:hep-ph/0111027

[12] E.E. Boos, Yu.A. Kubyshin, M.N. Smolyakov, I.P. Volobuev, Class.Quant.Grav. 19 (2002) 4591

[13] I.Ya. Aref'eva, M.G. Ivanov, W. Mück, K.S. Viswanathan, I.V. Volovich, Nucl.Phys. B590 (2000) 273

[14] M.N. Smolyakov, I.P. Volobuev, arXiv:hep-th/0208025

[15] M.N. Smolyakov, I.P. Volobuev, Theor.Math.Phys. 139(1) (2004) 458

[16] W.D. Goldberger, M.B. Wise, Phys.Rev.Lett. 83 (1999) 4922

[17] T. Gherghetta, M. Shaposhnikov, Phys.Rev.Lett. 85 (2000) 240

[18] T. Gherghetta, E. Roessl, M. Shaposhnikov, Phys.Lett. B491 (2000) 353

[19] P. Midodashvili, L. Midodashvili, Europhys.Lett. 65 (2004) 640

[20] I. Oda, Phys.Lett. B571 (2003) 235

[21] M. Gogberashvili, D. Singleton, Phys.Rev. D69 (2004) 026004

[22] T. Appelquist, H.C. Cheng, B.A. Dobrescu, Phys.Rev. D64 (2001) 035002

[23] N. Arkani-Hamed, M. Schmaltz, Phys.Rev. D61 (2000) 033005

[24] H. Georgi, A.K. Grant, G. Hailu, Phys.Rev. D63 (2001) 064027

[25] M. Gogberashvili, P. Midodashvili, Phys.Lett. B515 (2001) 447 
[26] M. Gogberashvili, P. Midodashvili, Europhys.Lett. 61 (2003) 308

[27] L.D. Landau, E.M. Lifshitz, The Classical Theory of Fields, Pergamon Press, Oxford, 1975

[28] T. Chiba, Phys.Lett. B575 (2003) 1

[29] W.D. Goldberger, M.B. Wise, Phys.Rev. D60 (1999) 107505

[30] H. Davoudiasl, J.L. Hewett, T.G. Rizzo, Phys.Lett. B473 (2000) 43

[31] J. Garriga, O. Pujolas, T. Tanaka, Nucl.Phys. B605 (2001) 192

[32] O. DeWolfe, D.Z. Freedman, S.S. Gubser, A. Karch, Phys.Rev. D62 (2000) 046008

[33] A. Kehagias, K. Tamvakis, Phys.Lett. B504 (2001) 38

[34] M.A. Luty, M. Porrati, R. Rattazzi, JHEP 0309 (2003) 029

[35] V.A. Rubakov, arXiv: hep-th/0303125 